\title{
Vol. 71, No. 3
}

In the report "Racial and Ethnic Disparities in Receipt of Medications for Treatment of COVID-19 - United States, March 2020-August 2021," on p. 98, in Table 1, under the "Inpatients with positive SARS-CoV-2 test result" column, the numbers and percentages for the following rows listed under "Medical conditions associated with high risk" should have read Anemia "28,645 (23.8)," Arrythmia "33,443 (27.8)," Asthma "14,542 (12.1)," COPD "13,447 (11.2)," Cancer "11,642 (9.7)," Chronic kidney disease "26,221 (21.8)," Chronic pulmonary disorders "28,994 (24.1)," Coagulopathy "18,908 (15.7)," Congestive heart failure "21,246 (17.7)," Coronary artery disease "25,308 (21.1)," Diabetes type 2 “41,888 (34.8)," Hypertension “69,671 (58.0)," Mental health disorders "23,857 (19.8)," Peripheral vascular disorders "14,484 (12.0)," and Severe obesity (BMI $\left.\geq 40 \mathrm{~kg} / \mathrm{m}^{2}\right)$ “17,716 (14.7)."

On p. 99, in Table 2, under the column heading, "Total no. eligible for treatment," the numbers for the row headings "Monoclonal antibodies (November 2020-August 2021): Ethnicity," should have read Non-Hispanic "387,403," and Hispanic "80,176," and the percentages in the same rows under the column "Total no. (\%) treated" should have read Non-Hispanic “(2.9)," and Hispanic “(1.3).” These corrections do not affect findings of this report. 